\title{
Enemigos naturales nativos de mosca blanca (Hemiptera: Aleyrodidae) en el cultivo de ejote francés en Chimaltenango, Guatemala
}

\author{
Native natural enemies of whitefly (Hemiptera: Aleyrodidae) \\ in French bean crop in Chimaltenango, Guatemala \\ Claudia E. Toledo-Perdomo \\ Centro Universitario de Santa Rosa, Cuilapa, Universidad de San Carlos de Guatemala, Guatemala
}

*Autor al que se dirige la correspondencia: toledo.perdomo@gmail.com

Recibido: 21 de junio 2019 / Revisión: 24 de septiembre 2019 / Aceptado: 07 de noviembre 2019

\section{Resumen}

E 1 complejo de mosca blanca (Hemiptera: Aleyrodidae) incluye algunas de las principales plagas del ejote francés (Phaseolus vulgaris L.). Dentro de las cuales, Bemisia tabaci es vector del virus del mosaico dorado que afecta la calidad y rendimiento del cultivo, con pérdidas hasta del $100 \%$ y un control difícil debido a la resistencia adquirida por las plagas hacia algunos agroquímicos. El ejote francés ocupa el segundo lugar entre de los productos no tradicionales de exportación de Guatemala. Su manejo agronómico ha sido principalmente a través del control químico, el cual afecta insectos y otros organismos que no son el objetivo del control, tales como: polinizadores, insectos benéficos, humanos y fauna silvestre. Los objetivos del estudio fueron: determinar la presencia de enemigos naturales nativos de la mosca blanca e identificar las especies de mosca blanca presentes en el cultivo del ejote francés en Chimaltenango. Para el estudio se establecieron cuatro parcelas de $300 \mathrm{~m}^{2}$, se realizaron muestreos semanales durante dos ciclos del cultivo. En cada parcela se muestrearon cinco sitios y en cada sitio cinco plantas. Las especies de parasitoides nativos encontrados fueron: Encarsia formosa Gahan, Eretmocerus eremicus Rose \& Zolnerowuch y Amitus fuscipennis MacGown \& Nebeker, la especie más abundante fue A. fuscipennis. Los depredadores identificados fueron Chrysoperla carnea (Stephens) e Hippodamia convergens Guerin-Meneville. La especie más abundante fue $H$. convergens. Estas especies podrían ser herramientas valiosas para ser empleadas en programas de control biológico, producciones orgánicas o en programas de manejo integrado de plagas.

Palabras claves: Trialeurodes vaporariorum, Bemisia tabaci, Phaseolus vulgaris, Amitus fuscipennis, Hippodamia convergens

\begin{abstract}
$\mathrm{T}$ The whitefly complex (Hemiptera: Aleyrodidae) includes some of the main pests of the French green bean (Phaseolus vulgaris L.). Among which, Bemisia tabaci is a vector of the golden mosaic virus that affects the quality and yield of the crop, with losses up to $100 \%$ and difficult control due to the resistance acquired by pests towards some agrochemicals. The French green bean ranks second among the non-traditional export products of Guatemala. Its agronomic management has been mainly through chemical control, which affects insects and other organisms that are not the objective of the control, such as: pollinators, beneficial insects, humans and wildlife. The objectives of the study were: to determine the presence of natural enemies native to the whitefly and identify the species of whitefly present in the cultivation of the French bean in Chimaltenango. For the study, four $300 \mathrm{~m}^{2}$ plots were established, weekly sampling was carried out during two crop cycles. Five sites were sampled on each plot and five plants on each site. The native parasitoid species found were: Encarsia formosa Gahan, Eretmocerus eremicus Rose \& Zolnerowuch and Amitus fuscipennis MacGown \& Nebeker, the most abundant species was A. fuscipennis. The predators identified were Chrysoperla carnea (Stephens) and Hippodamia convergens Guerin-Meneville. The most abundant species was $H$. convergens. These species could be valuable tools to be used in biological control programs, organic productions or in integrated pest management programs.
\end{abstract}

Keywords: Trialeurodes vaporariorum, Bemisia tabaci, Phaseolus vulgaris, Amitus fuscipennis, Hippodamia convergens 


\section{Introducción}

En Guatemala los cultivos de arvejas y otros vegetales generan alrededor de 45,000 empleos y más de 3.7 millones de jornales al año que se desempeñan en las prácticas agrícolas desde la siembra hasta la cosecha, además del procesamiento post-cosecha de estos productos. En el área de productos no tradicionales en Guatemala, estos contribuyen a un gran desarrollo que beneficia directamente a las familias del área rural, principalmente en las regiones del altiplano central, occidental y los departamentos de Alta Verapaz, Baja Verapaz y Jalapa (Asociación Guatemalteca de Exportadores, 2013).

Dentro de las principales plagas que atacan al cultivo del ejote francés están las moscas blancas Bemisia tabaci (Gennadius, 1889) y Trialeurodes vaporariorum (Westwood, 1856) que han sido desde las últimas décadas una importante plaga en los cultivos de frijol (Phaseolus vulgaris L.), chile (Capsicum annuиm L.), tomate (Solanum lycopersicum L.), y otras hortalizas, ocasionando grandes daños a los cultivos debido a que son vectores de varios virus. Siendo $B$. tabaci vector de los begomovirus y los crinivirus trasmitidos por Trialeurodes vaporariorum (Czosnek, Hariton-Shalev, Sobol, Gorovits, \& Ghanim, 2017; Wisler, Duffus, Liu, \& Li, 1998).

Las especies de mosca blanca B. tabaci y T. vaporariorum son plagas importantes en diversos cultivos. Están ampliamente distribuidas en regiones tropicales y subtropicales del mundo donde afectan más de 600 especies de plantas cultivadas y silvestres. Los daños que causan se deben tanto al efecto del insecto en las plantas atacadas y a su papel como vectores de enfermedades virales. En su alimentación, la mosca blanca provoca un debilitamiento en la planta por la extracción de nutrientes, afectan el crecimiento y producción del cultivo, desórdenes fisiológicos y la transmisión de virus (Cuéllar \& Morales, 2006; Morales, 2006). En el ejote francés los virus del mosaico dorado y el mosaico dorado amarillo causan pérdidas en rendimiento hasta del 100 \% (Cuéllar \& Morales, 2006; González Bez, Muñiz Cuenca, \& García Sánchez, 2002; Morales \& Anderson, 2001; Myartseva, Ruiz-Cancino, Coronado-Blanco, \& Corona-Lopez, 2010; Perring, 2001; Villar Sánchez, López Salinas, \& Acosta Gallegos, 2003).

Estudios realizados han demostrado que la mosca blanca biotipo Q se alimenta mejor que el biotipo B en plantas de tomate y algodón, aunque este segundo produce desórdenes fisiológicos en las plantas hospe- dantes. El biotipo B prefiere repollo y pepino (Liu et al., 2012). Los principales cultivos afectados por los begomovirus, los cuales son transmitidos por $B$. tabaci en América Latina, han sido: frijol común, tomate y los pimientos dulces y picantes. Las pérdidas económicas causadas por diferentes begomovirus en estos cultivos superan ampliamente las pérdidas económicas reportadas para otros cultivos. La importancia, distribución e incidencia de begomovirus en América Latina está directamente asociada a la distribución y dinámica poblacional de su vector B. tabaci y sus biotipos. A pesar de los esfuerzos para el control de la mosca blanca, esta plaga continúa causando pérdidas significativas de rendimiento y afectando a más regiones agrícolas de América Latina (Jones, 2003; Morales, 2006). Los begomovirus son trasmitidos únicamente por B. tabaci, biotipos A, B y Q. Los crinivirus pueden ser trasmitidos por especies de moscas blancas como Trialeurodes vaporariorum y T. abutiloneus. En la especie T. vaporariorum begomovirus puede ser ingerido por esta, pero es trasmitido exclusivamente por $B$. tabaci de manera persistente y circulativa (Czosnek et al., 2017; Orfanidou, Pappi, Efthimiou, Katis, \& Maliogka, 2016; Wisler et al., 1998).

El mal uso de los plaguicidas para el control de la mosca blanca ha dificultado su manejo (Campuzano-Martínez et al., 2010; Macías-Flores, Santillán-Ortega, Robles-Bermúdez, Ortíz-Cantón, \& Cambero-Campos, 2013; Naveen et al., 2017). Hay reportes de resistencia adquirida en la mosca blanca de moléculas de organofosforados, neonicotinoides, piretroides y organoclorados (Cuéllar \& Morales, 2006; García Valencia, Mesa Cobo, Estrada, \& Mena, 2013; Kandil, Saleh, El Dief, \& Farghaly, 2008) o disminución del efecto de estos para el control de la mosca blanca (Smith, Nagle, MacVean, \& McKenzie, 2016), esto podría ser la causa de que estas moléculas necesiten de dosis muy altas para su control, afectando a insectos benéficos en los cultivos, donde se ha observado en campo la disminución de la diversidad de enemigos naturales nativos de la mosca blanca en Chimaltenango.

Debido al posible impacto negativo de los agroquímicos sobre la fauna benéfica, seres humanos y fauna silvestre, es necesario realizar un diagnóstico inventario de los enemigos naturales nativos de la mosca blanca presentes en Chimaltenango, esto permitirá además de conocer las especies presentes, aportar información para tomar decisiones en futuros programas de control biológico e implementación de prácticas para la conservación y protección de estos enemigos naturales. 
El propósito de esta investigación fue realizar un inventario de los enemigos naturales nativos en las principales especies de mosca blanca presentes en el cultivo del ejote francés en Chimaltenango.

\section{Materiales y métodos}

La evaluación se realizó durante los meses de febrero a diciembre de 2018. Las parcelas se establecieron en el municipio de Parramos, departamento de Chimaltenango, Guatemala. Las parcelas estaban localizadas en las coordenadas latitud norte $14^{\circ} 34^{\prime} 58.3$ y longitud oeste $90^{\circ} 48^{\prime} 56.1$. El municipio se encuentra a una altitud de 1,760 msnm (Autoridad para el Manejo Sustentable de la Cuenca de Lago de Amatitlán, 2005). Las parcelas del ejote francés ( $P$. vulgaris var. Serengeti) de $300 \mathrm{~m}^{2}$ cada una, fueron seleccionadas en plantaciones comerciales para la exportación.

Haciendo una adaptación de las metodologías sugeridas para el estudio de las poblaciones de mosca blanca de Bueno, Cardona y Chacón (2005) y Bernal, Pesca, Rodríguez, Cantor y Cure (2008) se estableció la metodología para el muestreo de la presente evaluación. Se evaluaron en cada parcela cinco puntos seleccionados al azar y en cada punto cinco plantas al azar. El muestreo se realizó semanalmente durante dos ciclos del cultivo. Mediante boletas de campo se tomaron los datos de las muestras de las poblaciones de mosca blanca en cada tratamiento. Se evaluaron dos ciclos del cultivo, se tomaron datos climatológicos: temperatura, humedad relativa y precipitación. La temperatura fue tomada con un termómetro de pared analógico para temperatura ambiente con registro de máxima y escala en ${ }^{\circ} \mathrm{C}$. La precipitación pluvial se registró con un pluviómetro convencional plástico.

Los especímenes se colectaron manualmente, utilizando un pincel humedecido con etanol al $70 \%$, mediante una colecta manual. Para evitar el vuelo de los insectos presentes en las plantas de muestreo, estas fueron manipuladas cuidadosa y lentamente.

También se realizó una colecta con trampas que consistieron en platos amarillos, que contenían agua con jabón líquido. Estos fueron colocados en cada punto de muestreo de las parcelas y permanecieron en el sito de muestreo por $24 \mathrm{~h}$, posteriormente fueron revisados y se colectaron los especímenes presentes en las trampas. Todos los insectos colectados se preservaron en frascos de vidrio con etanol al $70 \%$ v:v.

\section{Identificación de especímenes colectados}

Los especímenes colectados se examinaron en un estereoscopio con aumento 40X y duplicador 2X. Para la identificación de las especies de mosca blanca que fueron colectadas se emplearon las claves taxonómicas de Caballero (1992) y Carapia-Ruiz y Castillo-Gutiérrez (2013) y descriptores de las especies de mosca blanca de Salas y Mendoza (1995).

Para la identificación de los enemigos naturales se emplearon las claves de Myartseva, Ruiz-Cancino, y Coronado-Blanco (2012), MacGown y Nebeker (1978), Viggiani y Evans (1992), Hernández-Suarez y colaboradores, (2003), Larson (2013), Penny, Tauber y Leon de (2000) y Stange (2018).

\section{Resultados}

Se determinaron dos especies de mosca blanca: $B$. tabaci y $T$. vaporariorum. Se colectaron en total 315 especímenes. La especie más abundante fue $T$. vaporariorum con un porcentaje del $95 \%$ (299 especímenes) del total de las especies de mosca blanca colectadas, y el $5 \%$ (16 especimies) correspondieron a la especie $B$. tabaci.

Los parasitoides colectados fueron: Encarsia formosa (Gaham,1924) (cuatro especímenes), Eretmocerus eremicus (Rose \& Zolnerowich,1997)) (siete especímenes) y Amitus fuscipennis) (576 especímenes) (MacGown \& Nebeker,1978). La especie más abundante fue $A$. fuscipennis, con el $98 \%$ de los especímenes de total de parasitoides colectados. Los depredadores identificados fueron Chrysoperla carnea (Stephens, 1836) (cuatro especímenes) e Hippodamia convergens (Guérin-Méneville, 1842) (23 especímenes). La especie más abundante de los depredadores fue $H$. convergens (Tabla 1 ), representando el $89 \%$ de depredadores de mosca blanca colectados.

Al relacionar la colecta de los parasitoides y depredadores con las condiciones climáticas de temperaturas máximas, mínimas y precipitación pluvial, se observó que $H$. convergens fue encontrada principalmente durante la época seca, durante el mes de abril y cuando empezaron las lluvias. En mayo, la temperatura máxima llegó hasta un grado mayor (23 ${ }^{\circ} \mathrm{C}$ ) que en el periodo de colecta de los otros enemigos naturales y la temperatura mínima fue constante para todas las colectas. El otro depredador, C. carnea y los parasitoides E. formosa, E. eremicus y A. fusci- 
Tabla 1

Enemigos naturales de las moscas blancas Bemisia tabaci y Trialeurodes vaporariorum y condiciones climáticas en el cultivo del ejote francés en el departamento de Chimaltenango, 2018

\begin{tabular}{|c|c|c|c|c|c|c|}
\hline & $\begin{array}{l}\text { Orden: } \\
\text { Familia }\end{array}$ & Especie & Cantidad & $\mathrm{T}^{\circ} \min$ & $\mathrm{T}^{\circ} \max$ & $\begin{array}{c}\mathrm{PP}(\mathrm{mm}) \\
\text { (acumulado) }\end{array}$ \\
\hline Parasitoide & $\begin{array}{l}\text { Hymenoptera: } \\
\text { Aphelinidae }\end{array}$ & Encarsia formosa & 4 & $10-11$ & 22 & 372 \\
\hline Parasitoide & $\begin{array}{l}\text { Hymenoptera: } \\
\text { Aphelinidae }\end{array}$ & Eretmocerus eremicus & 7 & $10-11$ & 22 & 372 \\
\hline Parasitoide & $\begin{array}{l}\text { Hymenoptera: } \\
\text { Platygasteridae }\end{array}$ & Amitus fuscipennis & 576 & $10-11$ & 22 & 372 \\
\hline Depredador & $\begin{array}{l}\text { Coleoptera: } \\
\text { Coccinellidae }\end{array}$ & Hippodamia convergens & 23 & $10-11$ & $22-23$ & 159 \\
\hline Depredador & $\begin{array}{l}\text { Neuroptera: } \\
\text { Chrysopidae }\end{array}$ & Chrysoperla carnea & 4 & $10-11$ & $21-22$ & 372 \\
\hline
\end{tabular}

pennis fueron colectados durante los meses de mayo y junio, cuando se presentó mayor precipitación pluvial (Figura 1).

\section{Discusión}

Los resultados obtenidos en este estudio sobre las especies presentes de mosca blanca en el cultivo de ejote francés son consistentes con lo que se reporta en la literatura científica. La mosca blanca B. tabaci se alimenta de más de 600 especies de plantas cultivadas y silvestres, es una especie ampliamente distribuida en regiones tropicales y subtropicales del mundo. Actualmente las dos especies de mayor importancia económica son B. tabaci y T. vaporariorum (Carapia Ruiz \& Castillo-Gutiérrez, 2013; Lorenção, Alves, Fugi, \& Matos, 2008), estas dos especies fueron identificadas en el cultivo del ejote francés en Chimaltenango.

Dentro del muestreo de mosca blanca la especie predominante fue $T$. vaporarioum, tomado en cuenta que $T$. vaporariorum no es vector de Begomovirus (Czosnek et al., 2017; Wisler et al., 1998) dentro de este grupo se encuentran los virus del mosaico dorado y el mosaico dorado amarillo, esto podría ser la causa de la baja presencia de estos virus durante los dos ciclos del ejote francés evaluados.

Conocer la especie predominante en una región, como en este caso, que fue T. vaporarioum, es relevante porque la presencia y abundancia de una especie podría influir en la presencia y abundancia de sus enemigos naturales. Otros factores que influyen en la abundancia de una especie es la disponibilidad de alimentos, en el caso de las especies fitófagas podría influir la relación planta-insecto.

Otros factores influyentes en la distribución geográfica de las poblaciones de insectos dependerán también en las condiciones climáticas que favorecen la reproducción de las especies. Por ejemplo, las temperaturas cálidas, humedad relativa moderada y precipitaciones relativamente bajas a moderadas (Castillo \& González, 2008). La planta hospedante es determinante para el comportamiento de la mosca blanca, como la oviposición, el desarrollo del insecto y la supervivencia. También hay estudios que han reportado la preferencia de las especies de mosca blanca a diversos cultivos (Greenberg, Jones, \& Tong-Xian, 2009; Shah, Zhang, \& Liu, 2015).

Las tres especies determinadas en este estudio: $A$. fuscipennis, E. formosa y E. eremicus, son parasitoides reportados para las especies de B. tabaci y $T$. vaporariorum (Gerling, Alomar, \& Arnó, 2001; Lahey, \& Stansly, 2015; Liu, Stansly, \& Gerling, 2015). Las Tres especies son parasitoides de las ninfas de mosca blanca, E. eremicus oviposita entre la ninfa de la mosca blanca y la superficie de la hoja, mientras que Encarsia y Amitus ovipositan dentro de la ninfa (Liu et al., 2015). Los cuatro instares ninfales pueden ser parasitados, aunque la preferencia por un instar particular difiere según la especie de parasitoide. En Amitus se ha reportado preferencia por ovipositar en los primeros dos instar (Drost, Qiu, Posthuman-Doodeman, \& van Lenteren, 1999). 


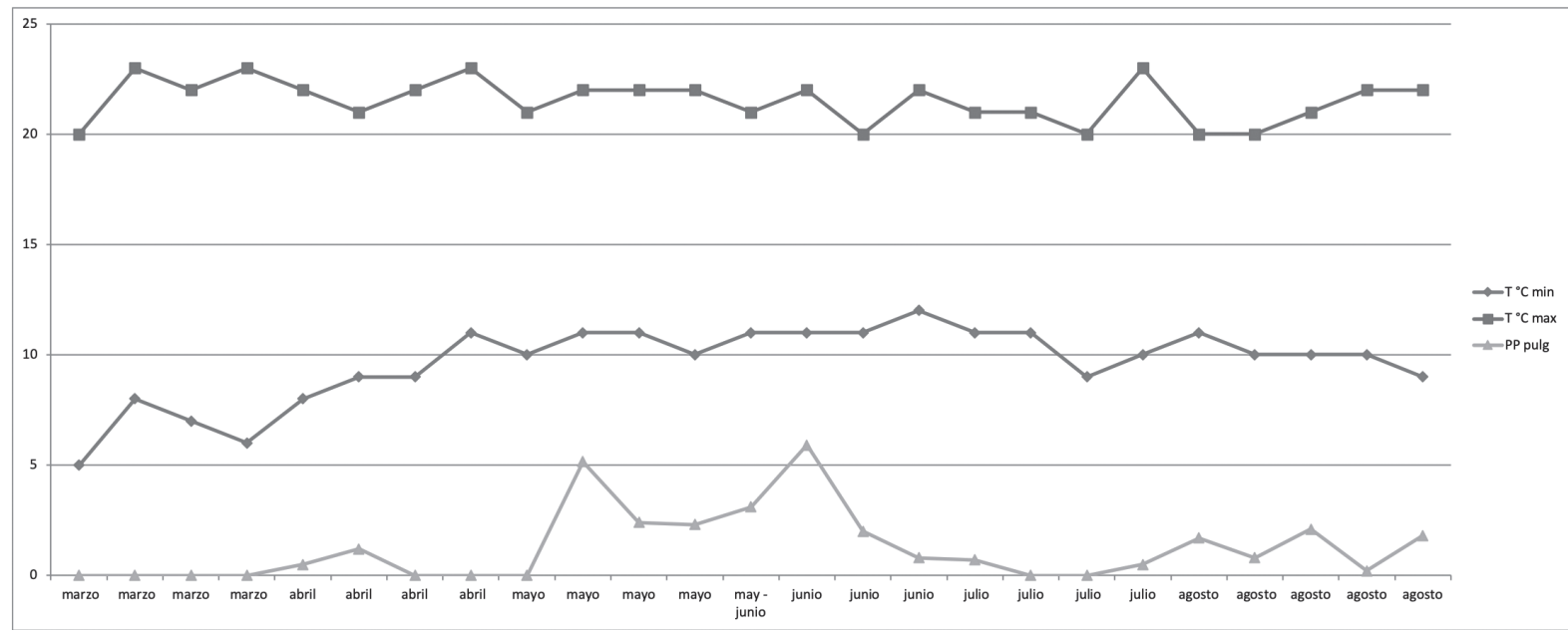

Figura 1. Temperaturas máximas y mínimas, precipitación pluvial durante el periodo de muestreo de los enemigos naturales de las moscas blancas Bemisia tabaci y Trialeurodes vaporariorum en el cultivo del ejote francés en el departamento de Chimaltenango, 2018.

El enemigo natural de la mosca blanca que presentó mayor abundancia en la colecta fue $A$. fuscipennis. Desde hace muchos años esta especie ha sido identificada en otros estudios realizados en el municipio de Acatenango, Chimaltenango a 2,400 msnm y también es reportada en Colombia, República Dominicana y México (MacGown \& Nebeker, 1978). El área de estudio de la presente investigación también fue en el departamento de Chimaltenango, municipio de Parramos, aproximadamente a $20 \mathrm{~km}$ de distancia entre estos municipios. Esto indica que, a pesar de las frecuentes aplicaciones de agroquímicos en estas zonas productoras de hortalizas en Guatemala, A. fuscipennis ha logrado mantener sus poblaciones. Tomando en cuenta esto, $A$. fuscipennis podría ser una herramienta para emplearla como control biológico dentro de un programa de manejo integrado de plagas en esta región para el control de la mosca blanca.

Además de la relación del parasitoide $A$. fuscipennis con la mosca blanca, también podría haber una relación y preferencia de este parasitoide con las plantas de $P$. vulgaris, en relación con otras plantas (Hernández-Suarez et al., 2003). Otras características de A. fuscipennis es su capacidad de dispersión, estudios realizados por Hernández y Manzano (2016) demostraron que su capacidad de dispersión supera los $12 \mathrm{~m}$ además de recibir la ayuda del viento, aprovechando su diminuto tamaño que contribuye en hacer más fácil su dispersión. Esta ventaja contribuye a que $A$. fuscipennis pueda llegar a otras regiones y cultivos.

A pesar que hay estudios que han reportado como algunos agroquímicos, especialmente los insecticidas pueden causar la mortalidad de parasitoides de mosca blanca (Sugiyama \& Saito, 2011). A. fuscipennis se encontró ampliamente distribuido en el cultivo del ejote francés en las parcelas evaluadas. Se encontró en una abundancia relacionada a la abundancia de $T$. vaporariorum.

Otro parasitoide encontrado fue E. formosa, esta especie es muy conocida porque ha sido empleada en programas de control biológico para el control del complejo mosca blanca B. tabaci y $T$. vaporariorum (Rodríguez, Rodríguez, Florido, \& Hernández, 1997).

Es posible que parte de la escasa población de $E$. formosa sea debido al efecto de los plaguicidas empleados para el manejo de plagas del ejote francés y otros cultivos vecinos como el brócoli (Brassica oleracea var. italica Plenck), arveja china y dulce (Pisum sativum L.), zanahoria (Daucus carota L.), zucchini (Cucurbita pepo L.), etc. Algunos estudios donde se reporta la alta susceptibilidad de $E$. formosa a moléculas de agroquímicos, indican una mortalidad superior del $98 \%$ después de las $6 \mathrm{~h}$ de haber aplicado el tratamiento químico de neonicotinoides como: el imidacloprid, acetamiprid, 
tiametoxan, las cuales son empleadas en la producción de hortalizas de la región. Otras moléculas empleadas en la región que también han sido reportadas como dañinas para $E$. formosa son: abamectina, cyantraniliprole y sulfoxaflor (Wang et al., 2019). El empleo de estos podría estar afectando las poblaciones nativas de $E$. formosa reduciendo considerablemente sus poblaciones. Otra posibilidad es que la abundancia relativa de las especies de enemigos naturales cambie con los meses del año o que tengan diferente distribución altitudinal, por lo que los resultados aquí obtenidos son válidos sólo para los meses y condiciones del estudio.

Encarsia y Eretmocerus son especies consideradas cosmopolitas, con amplia distribución a nivel mundial (Arnó, Gabarra, Liu, Simmons, \& Gerling, 2010), esta característica podría ser una ventaja para estas especies donde su amplio rango de distribución le permita una fácil adaptación a los distintos cambios climáticos y nichos ecológicos que se presentan en la región.

Tomado en cuenta que los parasitoides E. formo$s a$ y $E$. eremicus fueron encontrados en menor cantidad, es importante considerar principalmente que además del factor de toxicidad de agroquímicos sobre estos parasitoides, existe el factor de competencia entre parasitoides, que es importante considerar. Collier y Hunter (2001), estudiaron la interferencia de competición entre los parasitoides de la mosca blanca E. eremicus y E. sophia (Girault \& Dodd), a través de dos mecanismos que consisten en la usurpación del hospedero por multiparasitismo y a través de alimentarse del huésped, donde ambas especies podrían suprimir la progenie que está compitiendo con ellas. El estudio identificó a E. eremicus sobre E. sophia un multiparasitismo y a E. sophia sobre E. eremicus la combinación de multiparasitismo y alimentación del huésped en hospederos parasitados.

Estos factores son importantes considerarlos en un programa de manejo integrado de plagas, porque demuestra que es muy importante la elección del controlador biológico que se empleará y el efecto que puede causar cuando se utilizan otros agentes de control biológico al mismo tiempo.

La necesidad de implementar nuevas estrategias para el manejo de plagas y enfermedades es muy importante si se quiere entrar o permanecer en mercados de exportación, donde la demanda de la calidad e inocuidad de la cosecha son requisito indispensable. Por otra parte, también es importante orientar la producción agrícola hacia una producción sostenible y rentable para los productores de la región.
Con los resultados obtenidos se puede considerar la protección de especies nativas que pueden ser consideradas como control biológico para la mosca blanca en Chimaltenango. Es necesaria la reducción de la carga química en la producción agrícola y realizar prácticas culturales en los cultivos para la protección de los enemigos naturales.

\section{Agradecimiento}

Esta investigación fue cofinanciada por Digi-Usac-2018, Proyecto: 4.8.63.4.07. También se agradece a los productores de ejote francés del municipio de Parramos, Chimaltenango que colaboraron con la presente investigación.

\section{Referencias}

Arnó, J., Gabarra, R., Liu, T-X., Simmons, A. M., \& Gerling, D. (2010). Natural enemies of Bemisia tabaci: Predators and parasitoids. En P. Stansly, \& S. Naranjo (Eds.), Bemisia: Bionomics and Management of a Global Pest (pp. 385-421). Dordrecht, Netherlands: Springer.

Asociación Guatemalteca de Exportadores. (2013). Comité de Arvejas y vegetales. Recuperado de http://agexporthoy.export.com.gt/agexport/ en-los-ultimos-5-anos-guatemala-anualmenteha-exportado-80-millones-de-libras-de-arveja-yejote/

Autoridad para el Manejo Sustentable de la Cuenca de Lago de Amatitlán. (2005). Monografía del Municipio de Parramos. Guatemala: José Pineda Ibarra.

Bernal, L., Pesca, L., Rodríguez, D., Cantor, F., \& Cure, J. (2008). Plan de muestreo para Trialeurodes vaporariorum (Westwood) (Hemiptera: Aleyrodidae) en cultivos comerciales de tomate. Agronomía Colombiana, 26(2), 266-276.

Bueno, J. M., Cardona, C., \& Chacón, P. (2005). Fenología, distribución espacial y desarrollo de métodos de muestreo para Trialeurodes vaporariorum (Westwood) (Hemiptera:Aleyrodidade) en habichuela y frijol (Phaseolus vulgaris L.) Revista Colombiana de Entomología, 31(2), 161-170. 
Caballero, R. (1992). Whiteflies (Homopetera: Aleyrodidae) from Central America and Colombia field keys including slide-mouted pupal and field keys for identification, field characteristics, hosts, distribution, natural enemies and economic importance. (Tesis de maestría). Kansas State University, Kansas.

Campuzano-Martínez, A., Rodríguez-Maciel, J. C., Lagunes-Tejeda, A., Llanderal-Cázares, C., Teran-Vargas, A. P., Vera-Graziano, J., ... Silva-Aguayo, G. (2010). Aptitud biológica de poblaciones de Bemisia tabaci (Gennadius) biotipo B (Hemiptera: Aleyrodidae) con diferente susceptibilidad al insecticida thiametoxam. Neotropical Entomology, 39(3), 430-435. doi:10.1590-S1519566X2010000300018

Carapia Ruiz, V. E., \& Castillo-Gutiérrez, A. (2013). Estudio comparativo sobre la morfología de Trialeurodes vaporariroum (Westwood) y Bemisia tabaci (Gennadius) (Hemiptera: Aleyrodidae). Acta Zoológica Mexicana, 29, 178-193

Castillo, N., \& González, C. (2008). Comportamiento poblacional de insectos fitófagos en el unicultivo de frijol (Phaseolus vulgaris L.) y en la asociación con maíz (Zea mays L.). Revista de Protección Vegetal, 23(3), 154-159.

Collier, R. T., \& Hunter, M. S. (2001). Lethal interference competition in the whitefly parasitoids Eretmocerus eremicus and Encarsia sophia. Oecologia, 129, 147-154. doi:10.1007/s004420100706

Czosnek, H., Hariton-Shalev, A., Sobol, I., Gorovits, R., \& Ghanim, M. (2017) The incredible journey of begomoviruses in their whitefly vector. Viruses, 9(10), 273-291. doi:10.3390/v9100273

Cuéllar, M. E., \& Morales, F. J. (2006). La mosca blanca Bemisia tabaci (Gennadius) como plaga vectora de virus en fríjol común (Phaseolus vulgaris L.). Revista Colombiana de Entomología, 32(1), 1-9.

Drost, Y. C., Qiu, Y. T., Posthuma-Doodeman, C. J. A. M., \& van Lenteren, J. C. (1999). Life-history and oviposition behaviour of Amitus bennetti, a parasitoid of Bemisia argentifolii. Entomologia Experimentalis et Applicata, 90, 183-189. doi:10.1046/j.1570-7458.1999.00437.x

García Valencia, Y., Mesa Cobo, N. C., Estrada, E. I., \& Mena, Y. (2013). Estudio de la resistencia a Bemisia tabaci (Gennadius) (Hemiptera: Aleyrodidae) en germoplasma cultivado y silvestre de tomate (Solanum lycopersicum L.). Acta Agronómica, 62(4), 361-369.

Gerling, D., Alomar, O., \& Arnó, J. (2001). Biological control of Bemisia tabaci using predators and parasitoids. Crop Protection, 20(9), 779-799.

González Bez, M., Muñiz Cuenca, J., \& García Sánchez, E. (2002). Cultivos asociados y uso de arrope para el manejo de Bemisia tabaci y el virus del mosaico dorado en frijol. Manejo Integrado de Plagas y Agroecología, 66, 39-44.

Greenberg, S. M., Jones, W. A., \& Tong-Xian L. (2009). Tritrophic interactions among host plants, whiteflies and parasitoids. Southwestern Entomologist, 34(4), 431-445. doi:10.3958/059.034.0407

Hernández-Suarez, E., Carnero, A., Aguilar, A., Prinsloo, G., LaSalle, J., \& Polaszek, A. (2003). Parasitoids of whiteflies (Hymenoptera: Aphelinidae, Eulophidae, Platygastridae; Hemiptera: Aleyrodidae) from the Macaronesian archipelagos of the Canary Islands, Madeira and the Azores. Systematics and Biodiversity, 1(19), 55-108. doi:10.1017/ S1477200002001007

Hernández Mahecha, L. M., \& Manzano, M. R. (2016). Efecto del viento en la dispersión a corta distancia del parasitoide Amitus fuscipennis MacGown y Nebeker (Hymenoptera: Platygasteridae) en cultivos de fríjol y habichuela. Acta Agronómica, 6(1), 80-86. doi: 10.15446/acag.v65n1.48816

Jones, D. R. (2003). Plant viruses transmitted by whiteflies. European Journal of Plant Pathology, 109(3), 195-219. doi:10.1023/A:1022846630513

Kandil M. A., Saleh A. Y., El Dief. W. H., \& Farghaly, S. F. (2008). Resistance mechanisms of whitefly Bemisia tabaci (Homoptera: Aleyrodidae) to thiamethoxam and propenofos. Asian Journal of Biological Sciences, 1, 33-38. doi: 10.3923/ ajbs.2008.33.38

Larson, J. K. (2013). Key to lady beetles (Coleoptera: Coccinellidae) of Saskatchewan. Recuperado de http://www.entsocsask.ca/documents/insect_lists/ Coccinellidae_key.pdf

Lahey, Z., \& Stansly, P. (2015). An updated list of parasitoid Hymenoptera reared from the Bemisia tabaci species complex (Hemiptera: Aleyrodidae). Florida Entomologist, 98(2), 456-463. 
Liu, T-X., Stansly, P. A., \& Gerling, D. (2015). Whitefly parasitoids: Distribution, life history, bionomics, and utilization. Annual Review of Entomology, 60, 273-292. doi: 10.1146/annurev-ento-010814-021101

Liu, B., Yan, F., Chu, D., Pan, H., Jiao, X., Xie, W., ... Zang, Y. (2012). Difference in feeding behaviors of two invasive whiteflies on host plants with different suitability: Implication for competitive displacement. International Journal Sciences, 8(5), 697-706. doi: 10,7150/iijbs.4108

Lorenção, A. L., Alves, A. C., Fugi, C. G. Q., \& Matos, E. S. (2008). Outbreaks of Trialeurodes vaporariorum (West.) (Hemiptera: Aleyroridae) under field conditions in the state of São Paulo, Brazil. Neutropical Entomology, 37(1), 089-091.

MacGown, M. W., \& Nebeker T. E. (1978). Taxonomic review of Amitus (Hymenoptera: Proctotrupoidea, Platygastridae) of the western hemisphere. The Canadian Entomologist, 110(3), 275-283. doi: 10.4039/Ent110275-3

Macías-Flores, A., Santillán-Ortega, C., Robles-Bermudez, A., Ortíz-Cantón, M., \& Cambero-Campos, O. (2013). Selected events of resistance to insecticides in whiteflies (Hemiptera:Aleyrodidae) in the world. BioCiencias, 2(2), 4-16.

Morales, F. J. (2006). Hystory and current distribution of begomoviruses in Latin America. Advances in Virus Research, 67, 127-162. doi: 10.1016/S00653527(06)67004-8

Morales, F. J., \& Anderson, P. K. (2001). The emergence and dissemination of whitefly-transmitted geminiviruses in Latin America. Archives of Virology, 146(3), 415-441. doi:10.1016/S00653527(06)67004-8

Myartseva, S. N., Ruíz-Cancino, E., \& Coronado-Blanco, J. M. (2012). Aphelinidae (Hymenoptera: Chalcidoidea) de importancia agrícola en México. Revisión y claves. Tamaulipas, México: Universidad Autónoma de Tamaulipas.

Myartseva, S. N., Ruiz-Cancino, E., Coronado-Blanco, J. M., \& Corona-López, A. M. (2010). Especies de Encarsia (Hymenoptera: Aphelinidae) que parasitan Trialeurodes vaporariorum (Westwood) (Hemiptera: Aleyrodidae) en Tamaulipas y Morelos, México y descripción de una especie nueva. Dugesiana, 17(2), 129-135.
Naveen, N. C., Chaubey, R., Kumar, D., Rebijith, K. B., Rajagopal, R., Subrahmanyam, B., \& Subramanian, S. (2017). Insecticide resistance status in the whitefly, Bemisia tabaci genetic groups Asia-I, Asia-II-1 and Asia-II-7 on the Indian subcontinent. Cientific Reports, 7(40634), 1-15. doi: 10.1038/srep40634

Orfanidou, C. G., Pappi, P. G., Efthimiou, K. E., Katis, N. I., \& Maliogka, V. I. (2016). Transmission of Tomato chlorosis virus (ToCV) by Bemisia tabaci Biotype $\mathrm{Q}$ and evaluation of four weed species as viral sources. Plant Disease, 100(10), 2043-2049. doi: 10.1094/PDIS-01-16-0054-RE

Penny, N. D., Tauber, C. A., \& Leon de, C. A. T. (2000). A new species of Chrysopa from western North America with a key to North American species (Neuroptera: Chrysopidae). Annals of the Entomological Society of America, 93(4), 776784. doi:10.1603/0013-8746

Perring, T. M. (2001). The Bemisia tabaci species complex. Crop Protection, 20(9), S725-737. doi: 10.1016/S0261-2194(01)00109-0

Rodríguez, J. M., Rodríguez, R., Florido, A., \& Hernández, R. (1997). Integrated pest management on tomatoes in Gran Canaria. (Canary Islands). Bulletin de l'oilb-srop, 20(4), 39-44.

Shah, M. M., Zhang, S., \& Liu, T. (2015). Whitefly, host plant and parasitoid: A reviewon their Interactions. Asian Journal of Applied Science and Engineering, 4, 48-61.

Smith, H. A., Nagle, C. A., MacVean, C. A., \& McKenzie, C. L. (2016). Susceptibility of Bemisia tabaci MEAM1 (Hemiptera: Aleyrodidae) to imidacloprid, thiamethoxam, dinotefuran and flupyradifurone in south Florida. Insects, 7(57), 1-12. doi:10.3390/I.2016-7040057.

Salas, J., \& Mendoza, O. (1995). Biology of the Sweetpotato Whitefly (Homoptera: Aleyrodidae) on Tomato. The Florida Entomologist, 78(1), 154-160 doi: $10.2307 / 3495680$

Stange, L. (2018). Green lacewings (of Florida) (Insecta: Neuroptera: Chrysopidae). Recuperado de https://edis.ifas.ufl.edu/pdffiles/IN/IN96500.pdf

Sugiyama, K., Katayama, H., \& Saito, T. (2011). Effect of insecticides on the mortalities of three whitefly parasitoid species Eretmocerus mundus, Eretmocerus eremicus and Encarsia formosa $\mathrm{Hy}-$ 
menoptera: Aphelinidae. Applied Entomology and Zoology, 46(3), 311-317. doi: 10.1007/s13355011-0044-Z

Viggiani, G., \& Evans, G. A. (1992). Descriptions of three new species of Amitus Haldeman (Hymenoptera: Platygasteridae), parasitoids of known whiteflies from the New World. Bollettino del Laboratorio di Entomologia Agraria Filippo Silvestri Portici, 49, 189-194.

Villar Sánchez, B., López Salinas, E., \& Acosta Gallegos, J. (2003). Selección de genotipos de frijol por rendimiento y resistencia al mosaico dorado y suelos ácidos. Revista Fitotecnia Mexicana, 26(2), 109-114.
Wang, Z., Dai, P., Yang, X., Ruan, C. C., Biondi, A., Desneux, N., \& Zang, L. S. (2019). Selectivity of novel and traditional insecticides used for management of whiteflies on the parasitoid Encarsia formosa. Pest Management Science, 75(10), 2716-2724. doi:10.1002/ps.5380

Wisler, G. C., Duffus, J. E., Liu, H. -Y., \& Li, R. H. (1998). Ecology and epidemiology of whitefly-transmitted closteroviruses. Plant Disease, 82(3), 270-280. doi: 10.1094/PDIS.1998.82.3.270 